\title{
Cell-based 2-Step Scalar Deadzone Quantization for High Bit-Depth Hyperspectral Image Coding
}

\author{
Joan Bartrina-Rapesta and Francesc Aulí-Llinàs, Senior Member, IEEE
}

\begin{abstract}
Remote sensing images often need to be coded and/or transmitted with constrained computational resources. Among other features, such images commonly have high spatial, spectral, and bit-depth resolution, which may render difficult their handling. This paper introduces an embedded quantization scheme based on 2-step scalar deadzone quantization (2SDQ) that enhances the quality of transmitted images when coded with a constrained number of bits. The proposed scheme is devised for use in JPEG2000. It is named cell-based 2SDQ since it uses cells, i.e., small sets of wavelet coefficients within the codeblocks defined by JPEG2000. Cells permit a finer discrimination of coefficients in which to apply the proposed quantizer. Experimental results indicate that the proposed scheme is especially beneficial for high bit-depth hyperspectral images.
\end{abstract}

Index Terms-Embedded quantization, 2-step scalar deadzone quantization, high bit-depth images, JPEG2000.

\section{INTRODUCTION}

$\mathbf{T}$ HE bit-depth resolution of remote sensing images is often very high. Such images are produced by sensors with advanced dynamic range capabilities and very precise analogto-digital converters, and/or by post-acquisition processing techniques. Whereas natural images typically have a bit-depth of 8 bits per sample (bps), remote sensing images have 12 or even 16 bps. Some sensors with high bit-depth capacity are, for instance, the Airborne Visible/Infrared Imaging Spectrometer (AVIRIS) or the Hyperion. In addition to high bit-depth, most images employed in remote sensing also have high spatial resolution and are composed from tens to hundreds of spectral components. This makes their compression challenging.

To achieve high compression ratios and allow progressive transmission, high bit-depth hyperspectral images can be handled through lossy, or lossy-to-lossless, coding systems. A key feature of such systems is that they improve the quality of the whole image gradually as more data are transmitted. In wavelet-based compression schemes, this is achieved by an embedded quantizer together with a bitplane coding engine.

Embedded quantization splits the quantization index representing the magnitude of the wavelet coefficient in short words, possibly bits. Each word is a suffix of the previous (if any). Its transmission increases the precision with which the coefficient is reconstructed. The most popular scheme is uniform scalar deadzone quantization (USDQ) [1]. USDQ partitions the range of input values in intervals of the same size except for the

Copyright (c) 2015 IEEE. Personal use of this material is permitted. However, permission to use this material for any other purposes must be obtained from the IEEE by sending a request to pubs-permissions@ieee.org.

Dr. J. Bartrina-Rapesta and Dr. F. Aulí-Llinàs are with the Department of Information and Communications Engineering, Universitat Autònoma de Barcelona, Spain (phone: +34 935811861; e-mail: \{jbartrina | fauli\}@deic.uab.cat). This work has been partially supported by the Spanish Government (MINECO), by FEDER, and by the Catalan Government, under Grants RYC-2010-05671, TIN2012-38102-C03-03, and 2014SGR-691. interval that contains the zero, which is twice the size of the others. Intervals are assigned to quantization indices whose magnitude has a linear relation with values that they represent.

Bitplane coding engines transmit the binary representation of the quantization indices bit by bit, beginning by the most significant bit from all indices and finishing with the least significant. The collection of bits of all indices in the same binary position is called a bitplane. Bitplane coding together with USDQ achieves quality progressivity. Its implementation is not complicated, so such a scheme has been adopted in myriad wavelet-based coding systems [2], [3] and standards such as JPEG2000 (ISO/IEC 15444-1) or CCSDS 122.0-B-1.

Recently, embedded quantization for wavelet-transformed images has been studied from a more general point of view in [4]. The aim of [4] is to find schemes that achieve (near)optimal coding performance while requiring the minimum number of quantization stages. The best schemes found in [4] are embodied in the 2-step scalar deadzone quantization (2SDQ), which is applied in the framework of the JPEG2000 standard in [5]. The main idea behind 2SDQ is to use quantization intervals of two different sizes that are employed depending on the magnitude of the coefficient. Large-magnitude coefficients are sparse in wavelet subbands. To use larger quantization intervals for such coefficients does not affect coding performance significantly and reduces the number of quantization stages (or, equivalently, bitplanes) that are coded. Experimental results in [5] indicate that when the number of bits to represent the quantization indices is constrained, 2SDQ enhances the quality of the transmitted images notably as compared to a conventional JPEG2000 codec.

This work brings 2SDQ one step further. The conventional coding unit of JPEG2000 is the codeblock, i.e., a small set of adjacent wavelet coefficients within a subband. In [5], 2SDQ is applied considering the codeblock the smallest structure in which the quantization scheme can be applied. Herein, codeblocks are further divided in smaller units called cells. The selective application of 2SDQ in cells -instead of codeblocksprovides coding gains. The use of cells poses some difficulties such as the selection of the cells in which the scheme is applied, the parameters of the 2SDQ employed, and the effect in the rate-distortion optimization procedure. Our previous work [6] explores the use of the cell-based 2SDQ scheme for natural images. We found that the highest gains are obtained for high bit-depth hyperspectral images that have been transformed via the most effective transform strategies currently known [7]-[9], i.e., a 1D wavelet transform or a Karhunen-Loève transform in the spectral domain and then a $2 \mathrm{D}$ wavelet transform in the spatial domain $(1 \mathrm{D}+2 \mathrm{D}$ and KLT+2D, respectively). This works extends [6] by determining with more accuracy the parameters of 2SDQ and applying it 
to high bit-depth hyperspectral AVIRIS images.

The remainder of the paper is structured as follows. Section II briefly reviews USDQ and 2SDQ. Section III describes the proposed scheme and Section IV assesses its performance when coding AVIRIS images. The last section concludes this work with a brief summary.

\section{REVIEW OF USDQ AND 2SDQ}

Let $\omega$ be a coefficient of a wavelet-transformed image. USDQ quantizes $\omega$ according to

$$
v=\left\lfloor\frac{|\omega|}{\Delta}\right\rfloor,
$$

with $\lfloor\cdot\rfloor$ denoting the floor operation and $\Delta$ the step size of the quantization interval. Fig. 1(a) depicts the intervals produced by such a scheme in each quantization stage. $\mathcal{W}$ denotes the largest magnitude of the to-be-quantized coefficients. The first stage divides the range of input values into two intervals of equal size. This is produced when the most significant bit, say $b_{M-1}$, of quantization index $v$ is transmitted. $M$ denotes a sufficient number of bits to represent all coefficients. If $b_{M-1}=0$, the decoder reconstructs $\omega$ as $\hat{\omega}=0$. If $b_{M-1}=1$ the decoder reconstructs it as a number within the corresponding interval (i.e., $\hat{\omega} \in\left[2^{M-1}, 2^{M}\right)$ ). The typical mid-point reconstruction value is depicted in the figure with gray dots. The following quantization stages divide each previous interval into two sub-intervals of equal size, carrying out the coefficient reconstruction similarly as before. In general, the operation performed by the decoder is expressed as

$$
\hat{\omega}=\left\{\begin{array}{ll}
0 & \text { if } j^{\prime}>s \\
\operatorname{sign}(\omega)(\hat{v}+\delta) \Delta 2^{j^{\prime}} & \text { otherwise }
\end{array},\right.
$$

with $\hat{v}$ being all bits $\left\{b_{j}\right\}, M-1 \geq j \geq j^{\prime}$ transmitted for $v$ up to $j^{\prime}$, and $s$ denoting the position of the first non-zero bit of $v . b_{s}$ is called significant bit. $\delta$ controls the reconstruction point within the interval, which is commonly $\delta=0.5$. The sign of $\omega$ is transmitted just after $b_{s}$ so that the decoder can reconstruct the coefficient immediately after its significant bit.

Contrarily to USDQ, 2SDQ employs two step sizes, which are denoted by $\Delta_{L}$ and $\Delta_{H}$. As depicted in Fig. 1(b), $\Delta_{H}$ defines the intervals employed for large-magnitude coefficients, i.e., for $\omega \geq \alpha \mathcal{W}$, whereas $\Delta_{L}$ is employed for the remaining coefficients. The relation between these two step sizes is bounded by

$$
\Delta_{H}=\frac{(1-\alpha) \Delta_{L}}{\alpha},
$$

so that the number of intervals below and above $\alpha \mathcal{W}$ is the same. This assures that conventional bitplane coding strategies can be employed. The encoder quantizes the coefficients according to

$$
v^{\prime}= \begin{cases}\left\lfloor\frac{|\omega|}{\Delta_{L}}\right\rfloor & \text { if }|\omega|<\alpha \mathcal{W} \\ \left\lceil\frac{\alpha \mathcal{W}}{\Delta_{L}}\right\rfloor+\left\lfloor\frac{|\omega|-\alpha \mathcal{W}}{\Delta_{H}}\right\rfloor & \text { otherwise }\end{cases}
$$

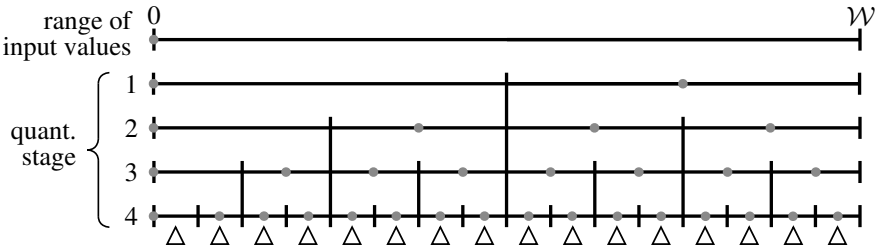

(a)

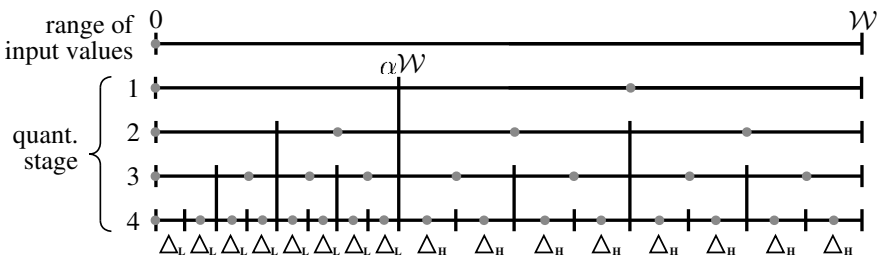

(b)

Fig. 1: Quantization intervals produced by (a) USDQ and (b) 2SDQ. Only the magnitude of the coefficient is depicted. Figure reproduced from [5].

where $\lceil\cdot\rceil$ denotes the ceiling operation. The decoder reconstructs the coefficients as

$$
\hat{\omega}^{\prime}=\left\{\begin{array}{c}
\text { if } j^{\prime}>s \\
\operatorname{sign}(\omega)\left(\hat{v}^{\prime}+\delta\right) \Delta_{L} 2^{j^{\prime}} \\
\text { if } j^{\prime} \leq s \text { and } \hat{v}^{\prime} 2^{j^{\prime}}<\left\lceil\frac{\alpha \mathcal{W}}{\Delta_{L}}\right\rceil \\
\operatorname{sign}(\omega)\left[\alpha \mathcal{W}+\left(\left(\hat{v}^{\prime}+\delta\right) 2^{j^{\prime}}-\left\lceil\frac{\alpha \mathcal{W}}{\Delta_{L}}\right\rceil\right) \Delta_{H}\right] \\
\text { otherwise. }
\end{array}\right.
$$

Note that the linear relation between $\omega$ and $v$ of the USDQ scheme does not hold in 2SDQ. When employing ratedistortion optimization methods, this compels to adjust the distortion decreases produced by the coding of each bit of $v^{\prime}$ (see below).

\section{CELl-BASED 2SDQ}

2SDQ can be applied in any wavelet-based coding system. It is mostly effective in systems in which the number of bits employed to represent the quantization indices is constrained. Such a situation may happen in devices in which the hardware processing units employed to code the image have data structures with a limited number of bits. Also, the proposed scheme helps to reduce the computational costs of the codec since fewer data are coded. In [5], 2SDQ is applied in JPEG2000 by modifying the quantization indices of selected codeblocks. Briefly described, let $M_{x}$ denote the number of magnitude bits needed to represent the quantization indices produced by conventional USDQ in codeblock $x$. Assume that the maximum number of magnitude bits that can be employed is $M^{\max }$. 2SDQ is applied in codeblocks whose $M_{x}>M^{\max }$. If $R_{x}=M_{x}-M^{\max }$, then the relation between the step sizes of USDQ and 2SDQ is 


$$
\Delta_{L}^{\prime}=\Delta \frac{\alpha 2^{M_{x}}}{2^{M^{\text {max }}-1}}=\Delta \alpha 2^{\mathcal{R}_{x}+1},
$$

and

$$
\Delta_{H}^{\prime}=\Delta(1-\alpha) 2^{\mathcal{R}_{x}+1} .
$$

Evidently, the codeblocks in which 2SDQ is applied must be signaled. The final codestream is not compliant with the standard, though it keeps all its features.

The main idea behind the proposed cell-based 2SDQ (CB2SDQ) is to apply 2SDQ in coding units smaller than the codeblock. This permits a more fine discrimination of the (sets of) coefficients in which such a scheme is beneficial. CB-2SDQ can be employed in the same scenarios as those previously discussed for $2 \mathrm{SDQ}$. The cell is defined as a square set of coefficients within the codeblock. Such a structure is also employed in [10] to accelerate the bitplane coding engine. Three aspects must be considered when employing CB-2SDQ. The first is that the application of 2SDQ is not dictated by $M_{x}$ but by the number of magnitude bits needed to represent all indices within the cell, denoted by $M_{c}$. 2SDQ is selectively applied in those cells in which $M_{c}>M^{\max }$. The step sizes employed in each cell are computed according to (6) and (7) but replacing $M_{x}$ by $M_{c}$ and $\mathcal{R}_{x}$ by $\mathcal{R}_{c}=M_{c}-M^{\max }$. Again, the cells in which 2SDQ is applied are signaled in the codestream.

The second aspect that the application of CB-2SDQ must consider is the value of $\alpha$ employed. In [5], $\alpha$ is roughly determined for a large variety of images and coding parameters as $\alpha=0.3$. We found that high bit-depth images such as those employed in the remote sensing field can benefit from a more finely tuned value of $\alpha$. Our objective is to transmit the image with the highest possible quality given $M^{\max }$ bits to represent quantization indices. The optimal $\alpha$ is that minimizing the distortion between the original and the reconstructed coefficients. When the distortion metric is mean squared error (MSE), this distortion is expressed as

$$
D=\sum_{\mathcal{C} \in c}\left(\omega[\mathcal{C}]-\hat{\omega}^{\prime}[\mathcal{C}]\right)^{2},
$$

with $\omega[\mathcal{C}]$ and $\hat{\omega}^{\prime}[\mathcal{C}]$ representing the original and the 2SDQ reconstructed coefficients within cell $c$, respectively. The distribution of coefficients within the cell determines the $\alpha$ that minimizes (8). When the cell contains few coefficients with large magnitudes, $\alpha$ will be lower than when the cell contains many coefficients with large magnitudes. The distribution of coefficients in wavelet subbands is commonly Laplace-like, though this may not hold in some detail subbands. High bitdepth resolution images may have cells with a probability density function (pdf) with longer and thinner tails than that achieved when the images have a more conventional bit-depth. The value of $\alpha$ for such images is therefore lower.

The optimization of $\alpha$ for a cell and a pre-defined $M^{\max }$ requires the conversion of the quantization indices for each value of $\alpha$ tested. This is not practical when the image
TABLE I: Empirically determined $\alpha$ for different transform strategies, resolution levels, and subbands (except for the LL subband, which is fixed to $\alpha=0.5$ ).

\begin{tabular}{|c|c|c|c|c|}
\cline { 2 - 5 } \multicolumn{1}{c|}{} & \multicolumn{2}{c|}{ 1D+2D } & \multicolumn{2}{c|}{ KLT+2D } \\
\hline dec. level & HL/LH & HH & HL/LH & HH \\
\hline 1 & 0.24 & 0.24 & 0.25 & 0.26 \\
\hline 2 & 0.24 & 0.24 & 0.25 & 0.26 \\
\hline 3 & 0.24 & 0.24 & 0.25 & 0.26 \\
\hline 4 & 0.26 & 0.26 & 0.25 & 0.27 \\
\hline 5 & 0.27 & 0.27 & 0.25 & 0.28 \\
\hline
\end{tabular}

is compressed onboard satellites or in sensors with limited computational resources. So the approach adopted herein is to model $\alpha$ for the type of image in hand. As studied in the literature [10], [11], all images of the same type (e.g., natural, AVIRIS, xRay,...) have a similar statistical behavior that can be exploited in probability models or rate-distortion optimization methods. This similarity is exploited herein to define a lookup table (LUT) for $\alpha$. To this end, an AVIRIS image is transformed through a $1 \mathrm{D}+2 \mathrm{D}$ and a KLT+2D strategy. Then, wavelet coefficients are conceptually partitioned in cells of size $16 \times 16,32 \times 32$, and $64 \times 64$. The quantization indices within each cell are converted to $2 \mathrm{SDQ}$ indices employing $M^{\max }=\{4,5,6,7,8\}$ and $\alpha=\{0.20,0.21,0.22, \ldots, 0.30\}$, computing $D$ for each different test. Experimental evidence suggests that $D$ is minimized for very similar values of $\alpha$ regardless of the size of the cell and the $M^{\max }$ employed, though different values are obtained depending on the wavelet subband. Table I reports the optimal $\alpha$ found for these AVIRIS images. HL and LH subbands have a similar pdf, so the values determined for both subbands are grouped. The higher the decomposition level, the higher the $\alpha$ since wavelet subbands at high decomposition levels concentrate more the energy of the image, which produces more coefficients of large magnitude. Similar results are obtained for other AVIRIS images.

The third aspect that is critical for the application of CB2SDQ is the rate-distortion optimization procedure. Commonly, JPEG2000 implementations construct the final codestream through Lagrange optimization. Key in such a method is to determine the rate-distortion slope of each bitstream segment that is put in the final codestream. The slope is the result of dividing the decrement in distortion by the increment in rate produced when the bitstream segment is transmitted. Typically, the distortion achieved at the end of coding pass $l$ is computed as the squared difference between the original and the reconstructed coefficients of codeblock $x$ according to

$D_{x}^{l} \approx G_{z}^{2} \Delta^{2} \sum_{\chi \in x}\left((v[\chi]+\delta)-\left\{\begin{array}{ll}0 & \text { if } j^{\prime}>s \\ (\hat{v}[\chi]+\delta) 2^{j^{\prime}} & \text { otherwise }\end{array}\right)^{2}\right.$,

where $G_{z}$ is the energy gain factor of subband $z$ to which the codeblock belongs. In the above expression $(v[\chi]+\delta)$ and $(\hat{v}[\chi]+\delta) 2^{j^{\prime}}$ represent the reconstructed coefficient when all bits, and when bits up to $j^{\prime}$, are transmitted, respectively. The distortion decrease that is produced when coding pass $l$ is transmitted is computed as $\triangle D_{x}^{l}=D_{x}^{l-1}-D_{x}^{l}$. 


$$
D_{x}^{l} \approx G_{z}^{2} \Delta^{2} \begin{cases}\beta_{L}^{\prime 2} \sum_{\mathcal{C} \in c}\left((v[\mathcal{C}]+\delta)-\left\{\begin{array}{ll}
0 & \text { if } j^{\prime}>s \\
(\hat{v}[\mathcal{C}]+\delta) 2^{j^{\prime}} & \text { otherwise }
\end{array}\right)^{2}\right. & \text { if } v[\mathcal{C}]<2^{M^{\text {max }}-1} \\
\beta_{H}^{\prime 2} \sum_{\mathcal{C} \in c}\left((v[\mathcal{C}]+\delta)-\left\{\begin{array}{ll}
0 & \text { if } j^{\prime}>s \\
(\hat{v}[\mathcal{C}]+\delta) 2^{j^{\prime}} & \text { otherwise }
\end{array}\right)^{2}\right. \text { otherwise } & \end{cases}
$$

Cells in which 2SDQ is employed must consider that the distortion decrements suffer a deviation with respect to cells in which USDQ is used. Due to the use of two step sizes, such a deviation is different depending on whether the index is larger or smaller than $2^{M^{\max }-1}$. The distortion decrease of indices that are smaller (larger) than $2^{M^{\max }-1}$ must be multiplied by $\beta_{L}=\alpha 2^{\mathcal{R}_{x}+1}\left(\beta_{H}=(\alpha 2 \ln 2-\alpha+1-\ln 2) 2^{\mathcal{R}_{x}+1}\right) \cdot \beta_{L}$ and $\beta_{H}$ are derived in [5]. Through $\beta_{L}$ and $\beta_{H}$, the distortion decrement produced in codeblocks that contain cells with $\mathrm{CB}$ 2SDQ indices can be finely adjusted by reformulating Eq. (9) in Eq. (10). $v[\mathcal{C}]$ denotes the quantization indices in cell $c$ and $\beta_{L}^{\prime}, \beta_{H}^{\prime}$ denote the deviations for cell $c$ computed with $\mathcal{R}_{c}$ instead of $\mathcal{R}_{x}$.

\section{EXPERIMENTAL RESULTS}

The proposed method is evaluated when coding four AVIRIS images belonging to Yellowstone scenes referred to as sc00, sc03, sc11, and sc18. These images have not been employed to generate the LUT for $\alpha$. They are $512 \times 677$ and have 224 components. Their bit-depth resolution is 16 bps. The aforementioned transform strategies (i.e., 1D+2D and KLT+2D) are employed to decorrelate spatial and spectral redundancy. JPEG2000 coding parameters are: 5 levels of irreversible 9/7 wavelet transform (both in the spatial and, when applicable, in the spectral domain), single quality layer, and no precincts.

The first set of experiments assess the image quality (in terms of signal to noise ratio (SNR)) and the coding rate (in terms of bps) when the images are coded using $M^{\max }=8,6$, and 4 bits. Table II reports the average results obtained for the four images. Four coding methods are shown. The first uses a JPEG2000 codec that transmits the $M^{\max }$ most significant bitplanes of each codeblock. Such a method yields the maximum SNR possible while maintaining compliance with the standard. The second method employs 2SDQ as it is formulated in [5]. As indicated in [5], the performance achieved by 2 SDQ is virtually the same as that of the practical GEQ described in [4], so the results reported for 2SDQ in this and following tests can be regarded as equivalent to those achieved by the practical GEQ. The third method is CB-2SDQ as it is described in the previous section. The last method is CB-2SDQ but it employs the optimal $\alpha$ for each cell instead of using the LUT defined in Table I. We recall the last method is not practical. It is reported herein for comparison purposes only. The codeblock size employed for JPEG2000 and 2SDQ is that indicated in the second row of each column. CB-2SDQ always utilizes a codeblock size of $64 \times 64$, whereas the cell size is that indicated in the second row of each column. For example, the results reported in the rightest column when
$M^{\max }=8$ utilize codeblocks of $16 \times 16$ for JPEG2000 and 2 SDQ and codeblocks of $64 \times 64$ and cells of $16 \times 16$ for CB2SDQ.

The results of Table II indicate that, in terms of SNR, CB2SDQ improves the results achieved by 2 SDQ. The gain varies depending on the codeblock size, cell size, and transform strategy, though they are generally between 0.1 to $0.7 \mathrm{~dB}$ higher. Compared to JPEG2000, the proposed method yields images of $2.8 \mathrm{~dB}$ higher, on average. The results of Table II also suggest that the model of $\alpha$ embodied in Table I works well in practice since the image quality achieved when the optimal $\alpha$ is employed is only slightly superior to that achieved by CB-2SDQ, except for a few cases. In terms of coding rate, the conventional JPEG2000 implementation achieves the lowest rates. Although all methods code the same number of bitplanes, the bitplanes in the conventional JPEG2000 implementation convey less information (and so the image has lower quality) than when using (CB-)2SDQ, which makes them more compressible. The coding rate differences between the remaining methods are not significant. We recall that the reported rate of the proposed method already includes the necessary ancillary information.

The progressive lossy performance achieved by the proposed method is appraised in Fig. 2 for one image of the corpus when $M^{\max }=6$ and the codeblock size is $32 \times 32$ for JPEG2000 and 2SDQ and $64 \times 64$ with cells of $32 \times 32$ for CB-2SDQ. Similar results are obtained for the other images, other values of $M^{\max }$, and other sizes of codeblocks and cells. Again, the performance achieved by the proposed method is higher than that of 2SDQ and slightly lower than that achieved with the implementation that computes the optimal $\alpha$. The proposed method yields a progressive coding performance significantly superior to that achieved by JPEG2000. Only at very low bitrates, the conventional JPEG2000 implementation achieves higher performance than that achieved by the remaining methods. This is because the highest bitplanes of all codeblocks can be transmitted earlier when 2SDQ is not employed since they contain less information. Also because the proposed method transmits more side information. At bitrates higher than those depicted in Fig. 2, the quality of the images is increased more progressively, though the differences among the methods reported is approximately maintained.

\section{CONClusions}

Embedded quantization is a technique employed in image coding systems to obtain quality progressive transmission. The most common approach is to use USDQ together with bitplane coding. Recent advances in the field have shown that the substitution of USDQ by a scheme that quantizes 
TABLE II: Evaluation of the SNR and coding rate (first and second value in each cell, respectively) achieved, in average by different methods when coding all the images of the corpus.

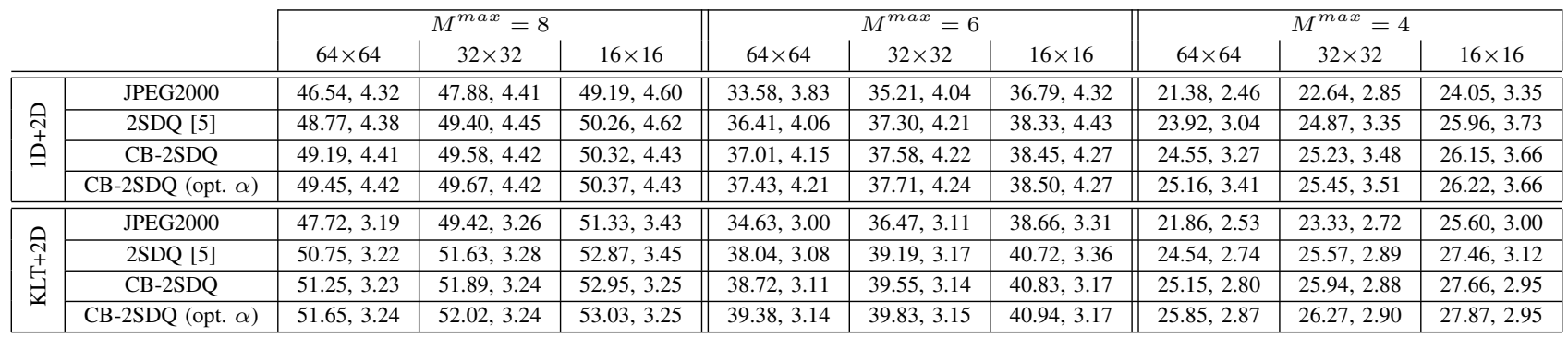

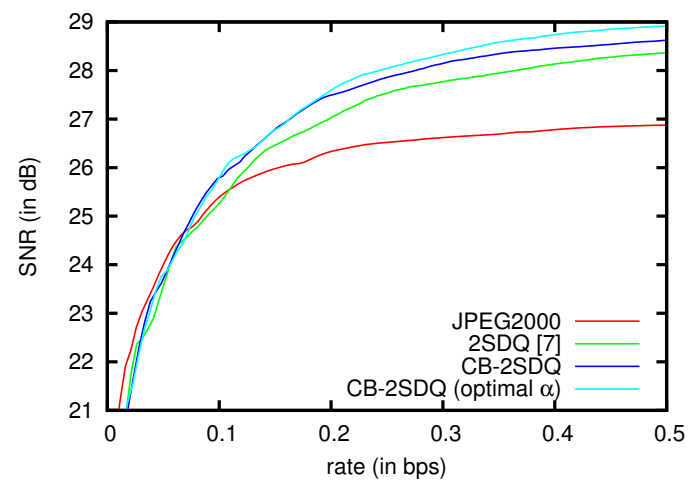

(a)

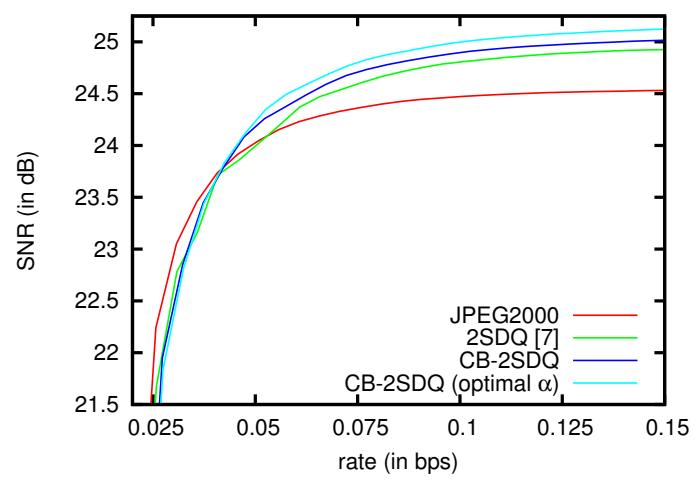

(b)

Fig. 2: Evaluation of progressive lossy coding performance for transform strategies (a) $1 \mathrm{D}+2 \mathrm{D}$ and (b) KLT+2D, for the sc11 image.

wavelet coefficients differently depending on their magnitude can produce images of higher quality at the same coding rates. 2SDQ is the first such a scheme introduced in the framework of JPEG2000. It is particularly efficient when the number of bits to represent quantization indices is constrained. This paper extends 2SDQ by selecting more finely the coefficients in which the quantizer is applied. Its main insight is to partition the JPEG2000 codeblock in smaller sets of coefficients called cells. Key to achieve competitive performance is to model the parameters of $2 \mathrm{SDQ}$ for their use in cells, and to adapt the ratedistortion optimization procedure of JPEG2000 accordingly.
The proposed cell-based 2SDQ (CB-2SDQ) scheme achieves high efficiency for images that have a high bit-depth resolution, like those employed in the remote sensing field. Experimental results for hyperspectral AVIRIS images indicate coding gains of more than $2 \mathrm{~dB}$ with respect to a conventional JPEG2000 implementation and between 0.1 to $0.5 \mathrm{~dB}$ with respect to $2 \mathrm{SDQ}$ at the same coding rates. The proposed method might also be introduced in other schemes for the coding of hyperspectral images such as [12], [13].

\section{REFERENCES}

[1] M. W. Marcellin, M. A. Lepley, A. Bilgin, T. J. Flohr, T. T. Chinen, and J. H. Kasner, "An overview of quantization in JPEG 2000," vol. 17, no. 1, pp. 73-84, Jan. 2002.

[2] A. Said and W. A. Pearlman, "A new, fast, and efficient image codec based on set partitioning in hierarchical trees," IEEE Trans. Circuits Syst. Video Technol., vol. 6, no. 3, pp. 243-250, Jun. 1996.

[3] W. A. Pearlman, A. Islam, N. Nagaraj, and A. Said, "Efficient, lowcomplexity image coding with a set-partitioning embedded block coder," IEEE Trans. Circuits Syst. Video Technol., vol. 14, no. 11, pp. 12191235, Nov. 2004.

[4] F. Auli-Llinas, "General embedded quantization for wavelet-based lossy image coding," IEEE Trans. Signal Process., vol. 61, no. 6, pp. 15611574, Mar. 2013.

[5] - "2-step scalar deadzone quantization for bitplane image coding," IEEE Trans. Image Process., vol. 22, no. 12, pp. 4678-4688, Dec. 2013.

[6] J. Bartrina-Rapesta, F. Auli-Llinas, I. Blanes, and J. Serra-Sagrista, "Cell-based 2-step scalar deadzone quantization for JPEG2000," in Proc. IEEE Data Compression Conference, Mar. 2014, pp. 143-152.

[7] B. Penna, T. Tillo, E. Magli, and G. Olmo, "Transform coding techniques for lossy hyperspectral data compression," IEEE Trans. Geosci. Remote Sens., vol. 45, no. 5, pp. 1408-1421, May 2007.

[8] J. E. Fowler and J. T. Rucker, "3D wavelet-based compression of hyperspectral imagery," in Hyperspectral Data Exploitation: Theory and Applications. John Wiley \& Sons, 2007, ch. 14, pp. 1-26.

[9] J. Serra-Sagrista and F. Auli-Llinas, "Remote sensing data compression," in Computational Intelligence For Remote Sensing. Germany: Springer Verlag, Jun. 2008, pp. 27-61, vol. 133 of studies in Computational Intelligence.

[10] F. Auli-Llinas and M. W. Marcellin, "Scanning order strategies for bitplane image coding," IEEE Trans. Image Process., vol. 21, no. 4, pp. 1920-1933, Apr. 2012.

[11] - "Stationary probability model for microscopic parallelism in JPEG2000," IEEE Trans. Multimedia, vol. 16, no. 4, pp. 960-970, Jun. 2014.

[12] Q. Du, W. Zhu, and J. E. Fowler, "Anomaly-based JPEG2000 compression of hyperspectral imagery," IEEE Geosci. Remote Sens. Lett., vol. 5, no. 4 , pp. $696-700$, Oct. 2008.

[13] X. Tang and W. Pearlman, "Scalable hyperspectral image coding," in Proc. IEEE International Conference on Acoustics, Speech, and Signal Processing, vol. 2, Mar. 2005, pp. 401-404. 